\title{
Assessment of Podcast-Enhanced Learning in Engineering Education
}

\author{
Kurtis G. Paterson, Ph.D., P.E. \\ Michigan Technological University \\ Department of Civil and Environmental Engineering
}

\begin{abstract}
Podcasting, a technology that lets anyone create and distribute radio- or TV-like shows over the Internet, is growing at an exponential rate. Current estimates suggest there are nearly 20 million podcasts, up from about 3 million just five months ago, and only 200 two years ago. While the technology offers exciting educational possibilities, university faculty are still ruminating about its use in learning.

This paper outlines the integration of enhanced-podcast episodes into a traditional Introduction to Environmental Engineering class. The objectives of this PodcastEnhanced Learning (PEL) research are to provide guidance for the creation of such podcasts, pedagogical evidence supporting best use of such podcasts within a university course setting, and student assessment of such efforts.
\end{abstract}

The enhanced-podcast creation process is a multi-step workflow. Major steps include media design, sound, video, and post-production. Free software tools were used in the production of the podcast episodes. Podcast design guidance will be shared in the paper, as will typical workflow resource requirements.

In the Introduction to Environmental Engineering class the enhanced-podcast was integrated into the class in three distinct methods for evaluation purposes: (1) as minidocumentaries for case study investigations, (2) as summaries of key concepts, and (3) as a lecture replacement. In-class time was used primarily for active learning. This approach provided for a mix of learning environments, and a platform to do controlled assessment of possible podcast implementations.

Student assessment of the PEL Environmental Engineering class was unanimously supportive. Students like the portability and on-demand nature of the podcast episodes. The more-balanced learning environment also was well received. Initial student assessment suggests enthusiasm for podcast episodes presenting case studies and key concepts, but not as a lecture replacement. In general these course modifications made the class enjoyable and memorable for the students. Considerable resource challenges must be confronted by the faculty and solved by university administrators before this educational approach can be widely adopted, unfortunately. 


\section{Background}

In Fall 2005 one in six college students took at least one course online. That's 850,000 more students than the year before, an increase of $40 \% .{ }^{1}$ This rapid growth is being facilitated by easier to use computer-based learning content creation tools, and a growing comfort of using information appliances.

Podcasting, a technology that lets anyone create and distribute radio- or TV-like shows over the Internet, is growing at an exponential rate. Current estimates suggest there are more than 100,000 podcast series, up from just a few hundred three years ago (see Figure 1). ${ }^{2}$ While the technology offers exciting educational possibilities, university faculty are still ruminating about its use in learning.

This paper introduces the integration of enhanced-podcast episodes into a traditional Introduction to Environmental Engineering class. The objectives of this PodcastEnhanced Learning (PEL) research were to provide:

- guidance for the creation of such podcasts

- pedagogical evidence supporting best use of such podcasts within a university course setting, and

- student assessment of such efforts.

\section{Podcast Design}

The enhanced-podcast creation process is a multi-step workflow. Major steps include media design, sound, video, and post-production. Free software tools were used in the production of twelve podcast episodes. Podcast design guidance and typical workflow resource requirements evolved during development of the early podcast episodes. Figure 2 shows the workflow developed for the enhanced podcast episodes for the Introduction to Environmental Engineering course. There were six major steps in the workflow, resulting in an 8-10 hour/episode average development time (above and beyond content creation). The entire workflow was executed with a production team of one person - the author.

\section{Step 1: Slides}

Despite the new delivery techniques that podcasting allows, content creation is still the most critical step; yet, podcasting places unique constraints on the content. Notably, in this project, the self-imposed podcast display constraint was a student watching an episode on a video iPod. As such, considerable design effort was required for the slides (in this case Keynote was used, but Powerpoint would be similarly constrained). Some important design findings include:

- Minimum font size: 72 point

- Minimum line thickness (for figures): 10 points

- Maximum lines of text: 4

- Photos: simple, close-ups only 
- Diagrams/graphs/schematics: simple, conceptual only

Additionally, high contrast and simple background/text designs work best. For most episodes created in this project, past class slides were re-designed according to these design rules. While this workflow phase is somewhat shorter starting from existing content, in many cases (particularly for figures) it became clear that creating new content following the above rules was the more expedient and effective way to go, rather than recrafting old materials. It is important to note that if existing content was not available for the episode, the development time for one episode approached twelve hours.

\section{Step 2: Voice-Annotated Slides}

One strength of the enhanced podcast is combining video and audio. The slides are the core video element, but the audio must be added somehow, and in a way that is synchronized with the slides. A simple but effective software package, Profcast, was used for this purpose. A simple studio is needed, essentially a computer with a microphone will suffice. Some important design findings include:

- An external microphone produces better results than a built-in one

- Conciseness is critical (file size is proportional to verbosity)

- Pausing between slides is fine, as the silence can be edited out

Additionally, rehearsing the spoken content is advised. While mistakes can be edited out, it is an immensely time-consuming process to locate the blunders in post-production.

\section{Step 3: Re-Sized Video File}

The output of the Profcast program is a video file (Quicktime or similar format). One issue with the current version was the restriction of the video file to a 180 by 180 pixel format - too small for implementation in the subsequent workflow steps. So the Profcast file was imported into Quicktime Pro, and resized to a 720 by 720 format. Profcast has released a new version (2.0) which corrects these issues.

\section{Step 4: Completed Video}

At this point in the workflow, the video file could be used as an adequate enhanced podcast. Steps 4 and 5 embellish the file with post-production steps to yield a slightly more professional looking episode. The resized video file was imported into iMovie so that opening and closing sequence (bumper and tail) could be added. These were used to provide a consistent and identifiable feel to the podcast series (much like most TV shows), and add any transition or special effects to the video. The closing sequence was also used to add additional commentary or guidance to the students (e.g. what to do next after watching the episode). The output file from iMovie (in Quicktime format) had complete video and voice-only audio.

\section{Step 5: Completed Audio}

For completeness, a simple audio track was added. To avoid any copyright issues, music was composed in the music-authoring software Garageband. Music was used to indicate changes in topics, or signal important points, much as sound effects are used in movies 
and television. Garageband also has the ability to create podcast-ready files, and was used for such purpose in this project.

Step 6: Podcast File

The finished podcast file was then uploaded to a webpage authoring application, iWeb, in which a class web site was constructed, one page hosting all the podcast episodes (Figure 3). One benefit of using iWeb is its ability to create the RSS (Really Simple Syndication) code. The RSS feed creates one of the advantages of podcasting, namely that users subscribe to the podcast series and their podcast viewing application (iTunes or similar) is automatically notified when a new episode is available. This eliminates the need for students to continually check for new episodes at the class web site.

\section{Pedagogical Design}

In the Introduction to Environmental Engineering class the enhanced-podcast was designed as a critical component to a balanced learning environment, not as a new delivery of ineffective teaching methods (e.g. creating hour-long lectures for an iPod). Core lecture segments were shifted to the podcast, allowing class time to be used for active learning exclusively. Podcasts have the advantages of pause and replay by the student, important for such reflective course elements like lecture. The podcast, and companion web site, directed the student to appropriate textbook material. Class time then built upon the podcast "lectures" with a mix of hands-on activities, problems, field trips, and discussion. This learning process is depicted in Figure 4. The PEL cycle, an adaptation of that by Kolb, ${ }^{3}$ creates an interdependence of the three learning elements: the podcasts, the textbook, and the in-class activities. The podcasts serve primarily to motivate learning by providing the core information, hopefully in an engaging way. Students are referred to the textbook for depth of knowledge. The in-class activities engage the students in applications of the material, then extend the knowledge to the next level with open-ended challenges. This approach provides a mix of learning environments, active and reflective, visual and verbal, sequential and global, and sensing and intuitive, hopefully yielding better learning and retention among the students. ${ }^{4,5,6}$ This PEL course was introduced on a small class of seven students in 2006, then revised for a class of seventeen in 2007.

Twelve episodes were created (see Table 1), and in 2007 another six were added from outside experts on other podcast series. The number of self-produced episodes depended primarily on development time available and an interest in examining student performance on subjects with and without a podcast. The target episode duration for this project was 10 minutes. This target was consistently missed. It was simply difficult to cull the critical material for each podcast episode to such a short duration. While the average episode duration is 35\% above this self-imposed duration and caused no issues for this class, consideration of length (and resultant file size) is critical should episodes be created for an entire class. Assuming 45 episodes for an entire semester-length course, and the averages found in this work, $10 \mathrm{hr}, 7 \mathrm{~min}, 30 \mathrm{sec}$ of total duration, and $828 \mathrm{MB}$ of files would be created for such a class. A reasonable concern for such an offering is the storage of such files on the student's information appliance (computer or iPod). Simply 
put, do they have the storage capacity? Additional, but infrequent, challenges could be placed on the podcast server if the class was large. In practice the storage and server issue seem to be largely insignificant to most users, thanks to rapid increases in information storage and processor speeds. Should a visionary university or academic program embark on a systematic adoption of PEL, these issues could be more pressing.

\section{Student Assessment}

Student assessment of the PEL Environmental Engineering class was unanimously supportive in both years. Students liked the portability and on-demand nature of the podcast episodes. The more-balanced learning environment also was well received. In general these course modifications made a traditionally loathed class enjoyable and memorable for the students.

A preliminary attempt to assess impacts on learning for subjects with and without podcasts revealed no learning benefit from the podcasts. The students do appreciated the focused content, liberation of the class from lecture, and portability of the materials though. Ideally, two sections with large enrollments would create a better assessment setting for retention objectives. A plan for this study is underway for Fall 2007.

As podcasting is new to education, the first assessment focused on application of such technology within university courses. With this feedback podcast episodes can be better designed and integrated to achieve retention goals and pedagogical strategies. At the conclusion of the course, a survey was used to assess student opinion of the PEL course, and the podcasts in particular. The results are summarized in Table 2.

Most students watched nearly all of the podcasts. Viewership increased in 2007 due to a stronger linkage to class materials. In 2006, the podcasts were merely provided as an additional resource. There was one student who watched two episodes only, she noted that she had repeated internet access issues from home. The students opted to view the podcasts on a web browser or via iTunes. These approaches allow viewing the podcast at much larger resolution. Interestingly, while several students had iPods, only one of the students owned a video iPod (out of both classes). While ownership may change, the iPod's resolution was used as the design constraint. It is clear that this important decision must be made for future projects: design for iPod or for a larger format (file size is strongly correlated to format size). Students watched the episodes almost one-and-a-half times, on average. Obviously, some students watched the episodes once, some twice. While several students watched all the episodes twice, no student watched an episode more than that. The students felt that a typical episode should last about 12-15 minutes, on average. This is encouraging considering that the average episode for the course average 13.5 minutes; however, it is not clear if this first exposure to podcasts conditioned the students to believe that an episode should be about this duration. Of note, none of the students had used any podcasts (enhanced, or standard) prior to this course in 2006, yet several students had at least heard of podcasts in 2007. Given four options (and the ability to provide "other" uses), the students clearly favored using podcasts in two ways: as done in this class, to present case studies, and (in 2007) to bring in outside 
experts. Case studies could be done as mini-documentaries and would be an excellent way to motivate students to learn material. The students were less interested in having one-hour long standard lectures or exam review sessions via podcasts. It is important to note that a couple students thought having podcasts for all these possibilities would be a great idea, but several clearly thought the same possibilities would be a poor use of podcasting. Lastly, the students were split as to which type of courses should have podcasting; a growing majority believe all classes should have podcasts, whereas slightly less felt introductory courses only should have them. Few students believed only upper diversion courses (junior, senior, graduate), or no courses should have podcasts. Students believe podcasting has educational value.

\section{Conclusions}

Enhanced-podcasting holds considerable promise as a modern tool for engineering education. The strengths of the technology play well to two educational approaches: (1) focused multimedia mini-lectures, which students can review at their discretion, and (2) case studies in the form of mini-documentaries. Both approaches serve well as motivational tools in the PEL learning cycle, and could resuscitate sagging student interest in reading textbooks. As such, they should be designed as one component in a balanced course offering, not as a class substitute.

The development time is a clear obstacle. In addition to the production and postproduction time requirements, there are considerable time requirements in mastering a diverse set of supporting skills: software applications, web authoring, script writing, videography, sound, and lighting to name but a few. While it is impressive that one person can do-it-all these days, such results will inevitably be inferior to those from a team of professionals, each focusing on their area of expertise. Therefore, considerable resource challenges must be confronted by the faculty and solved by university administrators before this educational approach can be widely adopted. 


\section{References}

1. Sloan Consortium. 2005. Growing by Degrees. http://www.sloan-

c.org/publications/survey/pdf/growing_by_degrees.pdf. Accessed: January 16, 2007.

2. BusinessWeek Online. 2005. Podcasting Explosion. http://www.businessweek.com/ technology/tech_stats/podcast050523.htm. Accessed: January 16, 2007.

3. Kolb, D.A. 1984. Experiential Learning: Experience as the Source of Learning and Development, Prentice-Hall.

4. Felder, R. 1996. Matters of Style, ASEE Prism, pp. 18-23.

5. Paterson, K.G. 2006a. Critical Connections: A First-Semester Course

in Environmental Engineering. 2006 ASEE National Conference Proceedings. 9 pp.

6. Paterson, K.G. 2006b. Building a Better Hybrid: Environmental Monitoring

and Measurement Analysis. 2006 ASEE National Conference Proceedings. 13 pp. 
Table 1. Introduction to Environmental Engineering enhanced-podcast episode topics, run durations, and file sizes

\begin{tabular}{clcc}
\hline Episode & Topic & Duration & File size \\
\hline 1 & Overview of environmental engineering & $7 \mathrm{~min}, 9 \mathrm{sec}$ & $11.5 \mathrm{MB}$ \\
2 & Mass balance & $11 \mathrm{~min}, 42 \mathrm{sec}$ & $16.4 \mathrm{MB}$ \\
3 & Completely mixed flow reactors & $17 \mathrm{~min}, 16 \mathrm{sec}$ & $23.5 \mathrm{MB}$ \\
4 & Plug flow reactors & $16 \mathrm{~min}, 28 \mathrm{sec}$ & $21.8 \mathrm{MB}$ \\
5 & Water resources & $16 \mathrm{~min}, 24 \mathrm{sec}$ & $21.5 \mathrm{MB}$ \\
6 & Groundwater resources & $12 \mathrm{~min}, 56 \mathrm{sec}$ & $17.1 \mathrm{MB}$ \\
7 & Wastewater fundamentals & $8 \mathrm{~min}, 16 \mathrm{sec}$ & $13.1 \mathrm{MB}$ \\
8 & Wastewater Pre-Treatment & $9 \mathrm{~min}, 53 \mathrm{sec}$ & $14.1 \mathrm{MB}$ \\
9 & Wastewater Primary Treatment & $11 \mathrm{~min}, 4 \mathrm{sec}$ & $15.3 \mathrm{MB}$ \\
10 & Wastewater Secondary Treatment & $26 \mathrm{~min}, 11 \mathrm{sec}$ & $31.2 \mathrm{MB}$ \\
11 & Wastewater Sludge Treatment & $15 \mathrm{~min}, 55 \mathrm{sec}$ & $22.4 \mathrm{MB}$ \\
12 & Wastewater Tertiary Treatment & $8 \mathrm{~min}, 38 \mathrm{sec}$ & $13.4 \mathrm{MB}$ \\
\hline Average & & $13 \mathrm{~min}, 30 \mathrm{sec}$ & $18.4 \mathrm{MB}$ \\
\hline Total & & $3 \mathrm{hr}, 41 \mathrm{~min}, 18 \mathrm{sec}$ & $220.8 \mathrm{MB}$ \\
\hline
\end{tabular}


Table 2. PEL Introduction to Environmental Engineering course student assessment summary results

\begin{tabular}{|c|c|c|}
\hline Question & $\begin{array}{c}\text { Average Result } \\
(2006, n=7)\end{array}$ & $\begin{array}{c}\text { Average Result } \\
(2007, \mathrm{n}=17)\end{array}$ \\
\hline $\begin{array}{l}\text { 1.) What fraction of the episodes did you } \\
\text { watch? }\end{array}$ & $75 \%$ & $95 \%$ \\
\hline $\begin{array}{l}\text { 2.) Which technology did you use to } \\
\text { watch the episodes? }\end{array}$ & $\begin{array}{c}\text { iTunes }=43 \% \\
\text { Browser }=57 \% \\
\text { Video iPod }=0 \%\end{array}$ & $\begin{array}{c}\text { iTunes }=29 \% \\
\text { Browser }=58 \% \\
\text { Video iPod }=4 \% \\
\text { Other }=8 \%\end{array}$ \\
\hline $\begin{array}{l}\text { 3.) How many times did you watch each } \\
\text { episode? } \\
\text { 4.) How long should an episode last? }\end{array}$ & $12.6 \mathrm{~min}$ & $14 \min$ \\
\hline $\begin{array}{l}\text { 5.) How should podcasts be used in } \\
\text { engineering education? Rate } 1-10 \text { ( } 1=\text { bad } \\
\text { idea, } 10=\text { great idea) }\end{array}$ & & \\
\hline a.) Like in this class & a.) 8.1 & a.) 8.4 \\
\hline b.) Hour-long lectures & b.) 5.6 & b.) 4.4 \\
\hline c.) Engineering case studies & c.) 8.0 & c.) 8.9 \\
\hline d.) Exam review sessions & d.) 5.7 & d.) 7.5 \\
\hline e.) Outside experts & e.) $n / a$ & e.) 8.5 \\
\hline
\end{tabular}

6.) Which classes should have podcasts available (choose one)?
a.) Upper division courses only
a.) 0
a.) $5 \%$
b.) Introductory courses only
b.) $43 \%$
b.) $19 \%$
c.) All
c.) $57 \%$
c.) $86 \%$
d.) None
d.) 0
d.) 0 


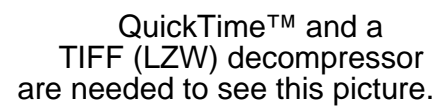

Figure 1. Estimates of podcast series available on the internet. 


$$
\begin{gathered}
\text { QuickTime }{ }^{\mathrm{TM}} \text { and a } \\
\text { TIFF (LZW) decompressor }
\end{gathered}
$$
are needed to see this picture.

Figure 2. Enhanced podcast episode workflow. The boxes denote the state of the podcast media during the workflow. 
QuickTime $^{\mathrm{TM}}$ and a

TIFF (LZW) decompressor

are needed to see this picture.

Figure 3. Screenshot of Introduction to Environmental Engineering podcast webpage. 


\section{QuickTime ${ }^{\mathrm{TM}}$ and a TIFF (LZW) decompressor are needed to see this picture.}

Figure 4. Podcast-Enhanced Learning (PEL) process. Font size of the three learning components (podcast, textbook, and in-class activities) shows their relative importance for each stage of the PEL cycle for a particular topic. 